\title{
Prolonged Electrocardiogram QRS Duration Independently Predicts Long-Term Mortality in Patients Hospitalized for Heart Failure With Preserved Systolic Function
}

\author{
SCOTT L. HUMMEL, MD, MS ${ }^{1}$, STEPHEN SKORCZ, MPH², and TODD M. KOELLING, MD, \\ FACC $^{1}$ \\ ${ }^{1}$ Division of Cardiovascular Medicine, Department of Internal Medicine, University of Michigan, \\ Ann Arbor, MI \\ ${ }^{2}$ Greater Flint Health Coalition, Flint, MI
}

\section{Abstract}

Background-Prolonged electrocardiogram (ECG) QRS duration ( $\geq 120 \mathrm{~ms}$ ) is a risk factor for death in systolic heart failure, but its effects in heart failure with preserved systolic function (HFPSF) have not been extensively studied. We hypothesized that prolonged ECG QRS duration would independently predict long-term mortality in hospitalized HFPSF patients.

\begin{abstract}
Methods and Results-We analyzed 872 HFPSF patients (defined as left ventricular ejection fraction 250\%) admitted to Michigan community hospitals between 2002 and 2004 and followed for a median of 660 days. We used Cox proportional hazards models to assess mortality hazard for prolonged QRS duration ( $\geq 120 \mathrm{~ms}$ ) on the last available predischarge ECG, first on a univariable basis and then after multivariable adjustment for other known risk factors. Prolonged QRS duration increased univariable all-cause mortality (HR 1.71; 95\% CI 1.33-2.19, $P<.001$ ) and after multivariable adjustment (HR 1.31; 95\% CI 1.01-1.71, $P=.04$ ). The univariable effect size was larger in younger patients. In multivariable models, there was no significant interaction between prolonged QRS and age, hypertension, or coronary artery disease status.
\end{abstract}

Conclusions-Prolonged QRS duration ( $\geq 120 \mathrm{~ms}$ ) on a predischarge ECG is an independent and consistent predictor of long-term mortality in hospitalized HFPSF patients.

\section{Keywords}

Diastolic heart failure; normal ejection fraction; outcomes; intraventricular conduction delay; elderly

\begin{abstract}
The incidence of heart failure with preserved systolic function (HFPSF) is increasing, ${ }^{1}$ particularly in the elderly. The prognosis of HFPSF remains poor, and more than half of patients are dead within 5 years of diagnosis. ${ }^{2}$ In systolic heart failure, many predictors of adverse prognosis have been defined, but in HFPSF less information is available. Although noncardiac comorbidities clearly impactlong-term HFPSF mortality, ${ }^{3}$ HFPSF patients also have a high risk for sudden cardiac and other cardiovascular death. ${ }^{4,5}$ In some cases, this risk may be related to known coronary artery disease, ${ }^{6,7}$ but half of hospitalized HFPSF patients do not carry this diagnosis. ${ }^{8}$ Additional information that could risk-stratify HFPSF
\end{abstract}


patients for mortality or suggest specific high-risk etiologic factors in an individual patient would be useful, particularly if it were easy to obtain.

Prolonged electrocardiogram (ECG) QRS duration is a risk factor for death in systolic heart failure. ${ }^{9-12}$ In systolic heart failure patients with QRS duration $\geq 120 \mathrm{~ms}$, treatment of associated systolic ventricular dyssynchrony ${ }^{13}$ with biventricular pacing decreases mortality and morbidity. ${ }^{14}$ In HFPSF patients, both prolonged QRS duration ${ }^{15}$ and ventricular dyssynchrony are common, ${ }^{16}$ but the significance of these findings is not yet clear in this population. ${ }^{17}$ Prior studies either were not intended to assess outcomes ${ }^{18}$ or investigated the association between prolonged QRS and adverse outcomes without adjusting for other important predictors. ${ }^{15,19}$

The physiology of HFPSF is heterogenous ${ }^{20}$ as are its underlying etiologies. Most commonly, HFPSF results from longstanding systemic hypertension and adverse cardiovascular remodeling. However, undiagnosed severe coronary disease and rarer causes of HFPSF such as restrictive cardiomyopathy, infiltrative diseases, and hypertrophic cardiomyopathy may both carry a poorer prognosis and be more likely to prolong intraventricular conduction. We hypothesized that prolonged ECG QRS duration would confer increased post-discharge mortality risk in hospitalized HFPSF patients, and analyzed its predictive power for death in a large community hospital sample of HFPSF inpatients assessed for mortality after a median of 22 months.

\section{Methods}

The Mid-Michigan Guidelines Applied in Practice - Heart Failure (GAP-HF) study was a collaborative effort between the Greater Flint Health Coalition, Michigan Peer Review Organization, and the Michigan Chapterof the American College of Cardiology to increase adherence to guideline-based inpatient heart failure performance measures. In 8 "intervention" hospitals, a multidisciplinary group developed performance measure targets and a guideline-based ${ }^{21}$ strategy to improve adherence. A group of 6 control hospitals, matched by number of beds, participated for comparison. Each hospital was assessed over 2 6-month intervals, 1 during a "baseline" period (October 1, 2002, through March 31, 2003) and again after the quality improvement program was adopted by the intervention hospitals (January 1, 2004, through June 30, 2004). Data on patient history, presenting symptoms and signs, rates of comorbidity, diagnostic studies, therapies, and inhospital outcomes were extracted through manual chart review by DynKePRO (York, PA), a Center for Medicare and Medicaid Services Clinical Data Abstraction Center with previously validated high reliability and accuracy. ${ }^{22}$ Mortality data on all patients was collected using the Social Security Death Index, with the assessment occurring greater than 1 year after discharge from the index hospitalization.

\section{Patient Selection}

We defined "preserved systolic function" as left ventricular ejection fraction (LVEF, obtained by echocardiography, nuclear scintigraphy, or contrast ventriculography) $\geq$ $50 \%{ }^{1,23-25}$ We excluded patients without numerical assessment of LVEF. Patients with known moderate or severe aortic or mitral stenosis were also excluded, because their heart failure may have been at least partially valvular in etiology. We excluded patients with no recorded ECG QRS duration, and excluded patients with previous placement of a permanent pacemaker or automated implantable cardiac defibrillator (as ECG tracings were unavailable for review to assess whether cardiac pacing could have artificially widened the QRS interval). We also removed HFPSF patients who had documented ventricular tachycardia on ECG, suffered a cardiac arrest, or died during the hospitalization, because widened ECG QRS duration in this subgroup could reflect arrhythmias. 


\section{Definitions of Variables Evaluated}

The ECG QRS duration was obtained from the ECG performed closest to hospital discharge, and was recorded based on the computerized interpretation of QRS width. ${ }^{26} \mathrm{We}$ dichotomized QRS duration at $120 \mathrm{~ms}$ ("prolonged QRS duration"). We calculated the body mass index (BMI) and analyzed it categorically ( $<25$; low or normal, $225-30$; overweight, and $330 \mathrm{~kg} / \mathrm{m}^{2}$ obese). We defined coronary artery disease status as presence on admission history or the occurrence of myocardial infarction or revascularization procedures during the index admission. Similarly, we defined atrial fibrillation as presence on admission history or ECG, or occurrence during the index admission.

We also calculated the 1-year Enhanced Feedback for Effective Cardiac Treatment (EFFECT) score, which predicts mortality at 1 year in community heart failure inpatients. ${ }^{3}$ The risk factors in this validated model include age, respiratory rate, admission systolic blood pressure, blood urea nitrogen, serum sodium, and admission history of stroke, dementia, chronic obstructive pulmonary disease, hepatic cirrhosis or cancer (in GAP+HF, defined as leukemia, lymphoma, or metastatic solid-organ cancer). The risk of all-cause 1year mortality increases with the 1-year EFFECT score.

\section{Statistical Analysis}

We performed all statistical analyses with STATA version 10.0 for Windows (StataCorp LP, College Station, TX). We report mean (SD) for continuous variables, and number (\%) for categorical variables. A P value of $<.05$ was required for statistical significance in all cases.

We grouped HFPSF patients by QRS duration $<120 \mathrm{~ms}$ or $\geq 120 \mathrm{~ms}$, and compared baseline clinical characteristics between these groups with 2-tailed independent sample $t$-tests for continuous variables and Pearson chi-square tests for categorical variables. We selected known or potential predictors of adverse events in HFPSF patients, and used Cox proportional hazards modeling to explore univariable associations of these variables and the QRS duration group with all-cause mortality in the entire HFPSF sample. We then used multivariable Cox proportional hazards modeling to explore the association of these variables and QRS duration with mortality.

In addition to QRS duration, the variables analyzed were: admission pulse rate, gender, race (Caucasian vs. non-Caucasian), BMI, history of heart failure, history of hypertension, coronary artery disease, atrial fibrillation, and the 1-year EFFECT score (see previous section for components). Because bradycardia with ventricular escape or undocumented ventricular tachycardia would widen the QRS interval and presumably also be associated with increased mortality, we repeated the analysis excluding patients who presented with pulse rate below 50 or greater than 99 beats $/ \mathrm{min}$. We also repeated the analysis including patients who died during the hospitalization $(n=19)$.

We summarized time-to-event outcomes with Kaplan-Meier survival curves, and differences between groups were summarized by the hazard ratio (HR) and a 95\% confidence interval (CI). We also performed exploratory analyses with univariable Cox models to assess the HR of prolonged QRS duration for death in the following subgroups: age (dichotomized at the median of 75 years), gender, race (white or other), BMI (obese or non-obese) history of coronary artery disease, preadmission history of heart failure, lowest quartile of admission systolic blood pressure $(<125 \mathrm{~mm} \mathrm{Hg}$ ), and admission renal function (dichotomized at the median estimated glomerular filtration rate of $\left.42 \mathrm{~mL} \cdot \mathrm{min} \cdot 1.73 \mathrm{~m}^{2}\right)$.

We added formal interaction terms to the multivariable Cox models for age above or below the median of 75 years, history of hypertension, and coronary artery disease. These specific factors were explored because we hypothesized that younger or non-hypertensive patients or 
those without known coronary artery disease might have undiagnosed, modifiable etiologies for HFPSF that could widen the QRS interval and affect prognosis. We constructed KaplanMeier survival curves for these subgroups as well.

\section{Results}

Of 3639 patients in GAP-HF, 2678 had numerical assessment of LVEF before or during the index admission. Of these, 2572 had assessment of ECG QRS duration during their hospitalization. A total of 963 of the remaining patients had LVEF $\geq 50 \%$, and after exclusion of subjects with known valvular disease $(n=75)$ or permanent pacemaker $(n=15)$ or automated implantable cardiac defibrillator $(n=2), 872$ comprised the HFPSF group for the present study. The baseline characteristics of the study patients are shown in Table 1, with HFPSF patients dichotomized by QRS duration and compared with 1012 systolic heart failure patients (defined as LVEF > 40\%, identical exclusion criteria to HFPSF group).

As shown by others, ${ }^{1,8,23}$ subjects with HFPSF were more obese, more often female, and had lower prevalence of known coronary artery disease and higher prevalence of hypertension than those with systolic heart failure. In GAP-HF HFPSF patients, the median EFFECT score of 97 placed the cohort in the "intermediate risk" group with a predicted 1year mortality of approximately $30 \%{ }^{3}$ The EFFECT model had reasonable performance (Cstatistic 0.70 in univariable logistic regression) for 1-year mortality in GAP-HF HFPSF patients. HFPSF patients with QRS duration $\geq 120 \mathrm{~ms}$ ( $22.4 \%$ of the cohort) were older, more likely to be male, and less obese than patients with QRS duration $<120 \mathrm{~ms}$. As expected, patients with prolonged QRS were more likely to have a history of coronary artery disease or atrial fibrillation, and additionally had higher mean EFFECT scores.

At a median follow-up of 660 days, observed mortality was $35.4 \%$ in the overall HFPSF cohort, but $31.5 \%$ in patients with QRS duration $<120 \mathrm{~ms}$ and $46.6 \%$ in patients with QRS duration $\geq 120 \mathrm{~ms}(P<.001)$. Significant univariable predictors of death included prolonged ECG QRS duration, non-Caucasian race, the EFFECT score, prior history of heart failure, atrial fibrillation, and BMI; univariable hazard ratios for all variables are shown in Table 2. We noted a strong univariable association between prolonged QRS duration and mortality (HR 1.70, 95\% CI 1.33-2.18, $P<.001$ ).

Kaplan-Meier survival curves for HFPSF patients with QRS duration $<120$ and $<120 \mathrm{~ms}$ are shown in Fig. 1, including the overall cohort and subgroups of age, history of hypertension, and history of coronary disease. A forest plot of hazard ratios with $95 \%$ confidence intervals and $P$ values for interaction terms for all analyzed subgroups is shown in Fig. 2. The unadjusted hazard ratio associated with prolonged QRS duration was generally consistent across the subgroups with the exception of patient age, where the hazard ratio for prolonged QRS was higher in younger patients, and BMI, where prolonged QRS trended toward higher hazard ratio in obese patients (Fig. 1,2).

Of the 872 HFPSF patients, 816 (93.6\%) had complete data on all analyzed variables and met all inclusion criteria. On multivariable Cox regression in this cohort, ECG QRS duration $\geq 120 \mathrm{~ms}$ remained an independent predictor of death (HR 1.31, 95\% CI 1.01-1.70, $P=.04$ ). Multivariable adjusted hazard ratios of the other analyzed factors for mortality are shown in Table 2. Exclusion of patients with pulse rate less than 50 or greater than 99 did not alter the results significantly (HR 1.34, 95\% CI 1.02-1.77, $P=.04$ ). Similarly, including patients who died in the hospital in the analyzed population $(n=19)$ did not change the results (HR $1.30,95 \%$ CI $1.00-1.68, P=.05)$. We did not find evidence of a significant interaction between prolonged QRS duration and history of hypertension or coronary artery disease. 
After multivariable adjustment, we also found no significant interaction between prolonged QRS duration and patient age.

\section{Discussion}

Hospitalized patients with HFPSF are at high risk for death, ${ }^{27}$ but predictors of mortality in this population are not as well defined as in systolic heart failure. We report a strong association between prolonged ECG QRS duration ( $\geq 120 \mathrm{~ms}$ ) and mortality in a moderately sized cohort of community hospital patients with HFPSF. In our study population, HFPSF patients with prolonged QRS duration were older and generally more ill than those with shorter QRS duration, highlighting the need to adjust for other mortality risk factors. To our knowledge, the present study is the first to confirm an association between prolonged ECG QRS duration and mortality in hospitalized HFPSF patients after controlling for multiple other known predictors of adverse outcomes in this population.

In systolic HF patients, prolonged ECG QRS duration is common and clearly a risk factor for mortality. In the Heart Failure Survival Score, commonly used to predict systolic heart failure mortality and select patients for cardiac transplantation, QRS duration $\geq 120 \mathrm{~ms}$ is one of the strongest predictors of death. ${ }^{10}$ More recent data confirm both the high prevalence of and adverse prognosis associated with prolonged QRS duration in this population. ${ }^{11}$ In systolic heart failure patients, QRS duration $\geq 120 \mathrm{~ms}$ is often associated with ventricular dyssynchrony, ${ }^{13,28}$ which impairs systolic and diastolic ventricular performance and is itself associated with increased mortality. ${ }^{29}$ Systolic heart failure patients with prolonged QRS randomized to cardiac resynchronization therapy with biventricular pacing devices have improvements in symptoms, quality of life, and long-term mortality. ${ }^{14}$

The significance of prolonged QRS duration in HFPSF is not as well understood. Few studies have estimated the prevalence of QRS duration $\geq 120 \mathrm{~ms}$ in this population; our study prevalence of $22 \%$ falls in the midrange of previous estimates ( $11 \%$ to $40 \%) .{ }^{15,18,19}$ One retrospective single-center study described increased combined mortality and hospitalization at six months in HFPSF patients (defined as LVEF $\geq 40 \%$ ) with QRS duration $\geq 120 \mathrm{~ms}$ when compared with those with shorter QRS duration, but adjusted only for age and did not include a formal survival analysis. ${ }^{15}$ Shenkman et al did not find a consistent association between QRS duration $\geq 120 \mathrm{~ms}$ and increased 5-year mortality in a larger cohort of HFPSF patients..$^{19}$ However, this database included mostly outpatients and the analysis adjusted only for age, gender, and race.

It is not clear whether the increased mortality in HFPSF patients with prolonged QRS is related to ventricular dyssynchrony, or whether cardiac resynchronization could decrease this risk. Recent work by Wang et al noted that $33 \%$ of HFPSF patients had systolic and $58 \%$ diastolic ventricular dyssynchrony, ${ }^{16}$ and others have related systolic dyssynchrony to heart failure symptoms in patients with normal systolic function. ${ }^{30}$ However, these studies were not intended to assess outcomes and did not report ECG QRS duration. Interestingly, $\mathrm{Yu}$ et al found that most HFPSF patients with systolic or diastolic dyssynchrony do not have prolonged QRS duration, although the overall prevalence of QRS duration $\geq 120 \mathrm{~ms}$ in this study was low. ${ }^{18}$

The true value of prolonged QRS duration in hospitalized HFPSF patients may lie not in suggesting dyssynchrony or therapeutic options, but rather in suggesting important undiagnosed causes of HFPSF. In patients with clinical HFPSF and known ischemic heart disease, the severity of coronary artery disease is a predictor of sudden cardiac death ${ }^{6}$ and overall mortality. ${ }^{7,31}$ However, large heart failure registries indicate that half of HFPSF 
patients do not carry a diagnosis of coronary artery disease on hospital admission for decompensated heart failure. ${ }^{8}$ As such, current guidelines for HFPSF advocate evaluation for ischemic heart disease. ${ }^{32}$

In GAP-HF, only $24 \%$ of HFPSF patients without acute coronary syndrome or known coronary artery disease had an invasive or noninvasive workup for ischemic heart disease during their hospital admission. Although it is likely that some patients had been evaluated before or were assessed after hospital discharge, it is also plausible that many HFPSF patients are never evaluated for coronary artery disease. As in other studies, ${ }^{8}$ GAP-HF HFPSF patients were predominantly elderly women, a group known to have lower likelihood of evaluation for coronary artery disease despite having equivalent prevalence to age-matched men. Prolonged QRS duration is a marker for long-term mortality in postmyocardial infarction patients ${ }^{33}$ as well as those referred to cardiac catheterization ${ }^{34}$ or nuclear exercise perfusion imaging ${ }^{35}$ for suspected coronary artery disease. Patients with QRS duration $\geq 120 \mathrm{~ms}$, particularly those with left bundle branch block, may also be more likely to have severe underlying coronary artery disease. ${ }^{36}$ In this study, we found that prolonged QRS was equally predictive of death in HFPSF patients with and without known coronary artery disease. We suggest that prolonged QRS duration should further prompt consideration of ischemic heart disease as the underlying etiology for decompensated HFPSF.

On the other hand, many patients with prolonged QRS duration do not have clinically significant coronary artery disease. In elderly patients, prolonged QRS may relate to particularly severe ventricular fibrosis and hypertrophy related to longstanding hypertension, ${ }^{37}$ or may reflect age-related degeneration of the conduction system. However, in GAP-HF the mortality hazard of QRS duration $\geq 120 \mathrm{~ms}$ had no clear relation to hypertension status, and as shown in Fig. 1 and 2, the mortality hazard of prolonged QRS duration was higher in younger patients before multivariable adjustment. Intriguingly, in 1 other prior study HFPSF patients with prolonged QRS duration had higher 6-month mortality than those with QRS duration $<120 \mathrm{~ms}$ despite being younger and having lower prevalence of known coronary artery disease; these subjects also had lower prevalence of hypertension. ${ }^{15}$

We hypothesize that the findings from Danciu et $\mathrm{al}^{15}$ and the present study may reflect an increased prevalence of rarer etiologies of HFPSF in patients with prolonged QRS duration. These causes may include non-hypertensive causes such as infiltrative diseases (eg, sarcoidosis or amyloidosis), restrictive cardiomyopathy, or hypertrophic cardiomyopathy, all of which may be associated with prolonged QRS duration. ${ }^{38-43}$ We cannot address this possibility in the present study without the necessary imaging and hemodynamic data. However, if present these etiologies would likely increase HFPSF mortality and could change therapy substantially, particularly in younger patients who are in the usual age group of incidence for these conditions. Diagnostic algorithms for HFPSF recommend consideration of these disorders, ${ }^{32}$ but it is not known how often this evaluation occurs. We propose that prolonged QRS duration should raise suspicion for "atypical" causes of HFPSF, particularly in younger or non-hypertensive patients.

Our findings should be interpreted in the context of several limitations. GAP-HF HFPSF patients with prolonged QRS duration were older, had higher prevalence of prior cardiovascular disease, and were generally more ill as evidenced by higher EFFECT scores. Although we adjusted for these factors in our model, it is possible that unmeasured correlates of prolonged QRS duration (eg, duration or severity of HFPSF prior to admission, undiagnosed coronary artery disease, myocardial infiltrative disease) affected outcomes. 
The data for QRS duration were based on computerized interpretation. Although ECG tracings were unavailable for review by the investigators, modern computer algorithms for measurement of QRS duration correlate highly with interpretations by experienced cardiologists ${ }^{26}$ and others have used this approach. ${ }^{19}$ In addition, we took several steps to exclude patients with devices or arrhythmias that could have artificially widened the QRS complex beyond its baseline. We did not have complete information on the morphology of intraventricular conduction delay (eg, bundle branch blocks) or on the ECG QT interval. We also did not have data on ventricular diastolic dysfunction, which is a known predictor of adverse outcomes in HFPSF, ${ }^{44}$ or ventricular dyssynchrony. Information on patient deaths was obtained from the Social Security Death Index, and no data on the causes of death were available.

\section{Conclusions}

In patients hospitalized for HFPSF, predischarge ECG QRS duration greater than $120 \mathrm{~ms}$ is an independent and consistent predictor of post-hospital death. In addition to its prognostic value, we propose that a prolonged QRS duration should prompt consideration of high-risk etiologies of HFPSF that are more likely to prolong intraventricular conduction. The relationships between prolonged ECG QRS duration, ventricular dyssynchrony, and outcomes in HFPSF patients remain incompletely defined and need to be studied further.

\section{Acknowledgments}

Funded by Greater Flint Health Coalition, AstraZeneca Pharmaceuticals, Pfizer, Inc., GlaxoSmithKline, Blue Cross Blue Shield of Michigan Foundation. S.L.H. is supported by an NIH T-32 research training grant, 5T32HL007853-10.

\section{References}

1. Owan TE, Hodge DO, Herges RM, Jacobsen SJ, Roger VL, Redfield MM. Trends in prevalence and outcome of heart failure with preserved ejection fraction [see comment]. N Engl J Med. 2006; 355:251-9. [PubMed: 16855265]

2. Tribouilloy C, Rusinaru D, Mahjoub H, Souliere V, Levy F, Peltier M, et al. Prognosis of heart failure with preserved ejection fraction: a 5 year prospective population-based study. Eur Heart J. 2008; 29:339-47. [PubMed: 18156618]

3. Lee DS, Austin PC, Rouleau JL, Liu PP, Naimark D, Tu JV. Predicting mortality among patients hospitalized for heart failure: derivation and validation of a clinical model. JAMA. 2003; 290:25817. [PubMed: 14625335]

4. Yusuf S, Pfeffer MA, Swedberg K, Granger CB, Held P, McMurray JJ, et al. Effects of candesartan in patients with chronic heart failure and preserved left-ventricular ejection fraction: the CHARMpreserved trial [see comment]. Lancet. 2003; 362:777-81. [PubMed: 13678871]

5. Ahmed A, Rich MW, Fleg JL, Zile MR, Young JB, Kitzman DW, et al. Effects of digoxin on morbidity and mortality in diastolic heart failure: the ancillary Digitalis Investigation Group trial. Circulation. 2006; 114:397-403. [PubMed: 16864724]

6. Al-Khatib SM, Shaw LK, O'Connor C, Kong M, Califf RM. Incidence and predictors of sudden cardiac death in patients with diastolic heart failure. J Cardiovasc Electrophysiol. 2007; 18:1231-5. [PubMed: 17883404]

7. Judge KW, Pawitan Y, Caldwell J, Gersh BJ, Kennedy JW. Congestive heart failure symptoms in patients with preserved left ventricular systolic function: analysis of the Cass registry. J Am Coll Cardiol. 1991; 18:377-82. [PubMed: 1856405]

8. Yancy CW, Lopatin M, Stevenson LW, De Marco T, Fonarow GC, et al. Clinical presentation, management, and in-hospital outcomes of patients admitted with acute decompensated heart failure with preserved systolic function: a report from the Acute Decompensated HEart failure national REgistry (ADHERE) database. J Am Coll Cardiol. 2006; 47:76-84. [PubMed: 16386668] 
9. Baldasseroni S, Opasich C, Gorini M, Lucci D, Marchionni N, Marini M, et al. Left bundle-branch block is associated with increased 1-year sudden and total mortality rate in 5517 outpatients with congestive heart failure: a report from the Italian network on congestive heart failure. Am Heart J. 2002; 143:398-405. [PubMed: 11868043]

10. Aaronson KD, Schwartz JS, Chen TM, Wong KL, Goin JE, Mancini DM. Development and prospective validation of a clinical index to predict survival in ambulatory patients referred for cardiac transplant evaluation. Circulation. 1997; 95:2660-7. [PubMed: 9193435]

11. Wang NC, Maggioni AP, Konstam MA, Zannad F, Krasa HB, Burnett JC Jr, et al. Clinical implications of QRS duration in patients hospitalized with worsening heart failure and reduced left ventricular ejection fraction. JAMA. 2008; 299:2656-66. [PubMed: 18544725]

12. Levy WC, Mozaffarian D, Linker DT, Sutradhar SC, Anker SD, Cropp AB, et al. The Seattle Heart Failure Model: prediction of survival in heart failure. Circulation. 2006; 113:1424-33. [PubMed: 16534009]

13. Bleeker GB, Schalij MJ, Molhoek SG, Verwey HF, Holman ER, Boersma E, et al. Relationship between QRS duration and left ventricular dyssynchrony in patients with end-stage heart failure. $\mathrm{J}$ Cardiovasc Electrophysiol. 2004; 15:544-9. [PubMed: 15149423]

14. Cleland JG, Daubert JC, Erdmann E, Freemantle N, Gras D, Kappenberger L, et al. The effect of cardiac resynchronization on morbidity and mortality in heart failure. N Engl J Med. 2005; 352:1539-49. [PubMed: 15753115]

15. Danciu SC, Gonzalez J, Gandhi N, Sadhu S, Herrera CJ, Kehoe R. Comparison of six-month outcomes and hospitalization rates in heart failure patients with and without preserved left ventricular ejection fraction and with and without intraventricular conduction defect. Am J Cardiol. 2006; 97:256-9. [PubMed: 16442374]

16. Wang J, Kurrelmeyer KM, Torre-Amione G, Nagueh SF. Systolic and diastolic dyssynchrony in patients with diastolic heart failure and the effect of medical therapy. J Am Coll Cardiol. 2007; 49:88-96. [PubMed: 17207727]

17. Kass DA. An epidemic of dyssynchrony: but what does it mean? J Am Coll Cardiol. 2008; 51:127. [PubMed: 18174030]

18. Yu C-M, Zhang Q, Yip GWK, Lee P-W, Kum LCC, Lam Y-Y, et al. Diastolic and systolic asynchrony in patients with diastolic heart failure: a common but ignored condition [see comment]. J Am Coll Cardiol. 2007; 49:97-105. [PubMed: 17207728]

19. Shenkman HJ, Pampati V, Khandelwal AK, McKinnon J, Nori D, Kaatz S, et al. Congestive heart failure and qrs duration: establishing prognosis study. Chest. 2002; 122:528-34. [PubMed: 12171827]

20. Maurer MS, King DL, El-Khoury Rumbarger L, Packer M, Burkhoff D. Left heart failure with a normal ejection fraction: identification of different pathophysiologic mechanisms. J Card Fail. 2005; 11:177-87. [PubMed: 15812744]

21. Hunt SA, Baker DW, Chin MH, Cinquegrani MP, Feldman AM, Francis GS, et al. ACC/AHA guidelines for the evaluation and management of chronic heart failure in the adult: executive summary. A report of the American College of Cardiology/American Heart Association task force on practice guidelines (committee to revise the 1995 guidelines for the evaluation and management of heart failure). J Am Coll Cardiol. 2001; 38:2101-13. [PubMed: 11738322]

22. Eagle KA, Montoye CK, Riba AL, DeFranco AC, Parrish R, Skorcz S, et al. Guideline-based standardized care is associated with substantially lower mortality in medicare patients with acute myocardial infarction: the American College of Cardiology's Guidelines Applied in Practice (GAP) projects in Michigan. J Am Coll Cardiol. 2005; 46:1242-8. [PubMed: 16198838]

23. Bhatia RS, Tu JV, Lee DS, Austin PC, Fang J, Haouzi A, et al. Outcome of heart failure with preserved ejection fraction in a population-based study. N Engl J Med. 2006; 355:260-9. [PubMed: 16855266]

24. Vasan RS, Levy D. Defining diastolic heart failure: a call for standardized diagnostic criteria [see comment]. Circulation. 2000; 101:2118-21. [PubMed: 10790356]

25. Yturralde RF, Gaasch WH. Diagnostic criteria for diastolic heart failure. Prog Cardiovasc Dis. 2005; 47:314-9. [PubMed: 16003646] 
26. Murkofsky RL, Dangas G, Diamond JA, Mehta D, Schaffer A, Ambrose JA. A prolonged qrs duration on surface electrocardiogram is a specific indicator of left ventricular dysfunction. $\mathrm{J}$ Am Coll Cardiol. 1998; 32:476-82. [PubMed: 9708478]

27. Gheorghiade M, Abraham WT, Albert NM, Greenberg BH, O'Connor CM, She L, et al. Systolic blood pressure at admission, clinical characteristics, and outcomes in patients hospitalized with acute heart failure [see comment]. JAMA. 2006; 296:2217-26. [PubMed: 17090768]

28. Ghio S, Freemantle N, Serio A, Magrini G, Scelsi L, Pasotti M, et al. Baseline echocardiographic characteristics of heart failure patients enrolled in a large European multicentre trial (CARE-HF; CArdiac RE-synchronisation Heart Failure study). Eur J Echocardiogr. 2006; 7:373-8. [PubMed: 16337208]

29. Cho G-Y, Song J-K, Park W-J, Han S-W, Choi S-H, Doo Y-C, et al. Mechanical dyssynchrony assessed by tissue Doppler imaging is a powerful predictor of mortality in congestive heart failure with normal QRS duration. J Am Coll Cardiol. 2005; 46:2237-43. [PubMed: 16360052]

30. Wang YC, Hwang JJ, Lai LP, Tsai CT, Lin LC, Katra R, et al. Coexistence and exercise exacerbation of intraleft ventricular contractile dyssynchrony in hypertensive patients with diastolic heart failure. Am Heart J. 2007; 154:278-84. [PubMed: 17643577]

31. O'Connor CM, Gattis WA, Shaw L, Cuffe MS, Califf RM. Clinical characteristics and long-term outcomes of patients with heart failure and preserved systolic function. Am J Cardiol. 2000; 86:863-7. [PubMed: 11024402]

32. Heart Failure Society of America. Executive summary: HFSA 2006 comprehensive heart failure practice guideline. J Card Fail. 2006; 12:10-38. [PubMed: 16500578]

33. Yerra L, Anavekar N, Skali H, Zelenkofske S, Velazquez E, McMurray J, et al. Association of QRS duration and outcomes after myocardial infarction: the VALIANT trial. Heart Rhythm. 2006; 3:313-6. [PubMed: 16500304]

34. Triola B, Olson MB, Reis SE, Rautaharju P, Merz CNB, Kelsey SF, et al. Electrocardiographic predictors of cardiovascular outcome in women: the National Heart, Lung, and Blood Institutesponsored Women's Ischemia Syndrome Evaluation (WISE) study. J Am Coll Cardiol. 2005; 46:51-6. [PubMed: 15992635]

35. Hesse B, Diaz LA, Snader CE, Blackstone EH, Lauer MS. Complete bundle branch block as an independent predictor of all-cause mortality: report of 7,073 patients referred for nuclear exercise testing. Am J Med. 2001; 110:253-9. [PubMed: 11239842]

36. Jain AC, Mehta MC. Etiologies of left bundle branch block and correlations with hemodynamic and angiographic findings. Am J Cardiol. 2003; 91:1375-8. [PubMed: 12767441]

37. Dhingra R, Ho Nam B, Benjamin EJ, Wang TJ, Larson MG, D'Agostino SRB, et al. Crosssectional relations of electrocardiographic QRS duration to left ventricular dimensions: the Framingham Heart Study. J Am Coll Cardiol. 2005; 45:685-9. [PubMed: 15734611]

38. Sekiguchi M, Yazaki Y, Isobe M, Hiroe M. Cardiac sarcoidosis: diagnostic, prognostic, and therapeutic considerations. Cardiovasc Drugs Ther. 1996; 10:495-510. [PubMed: 8950063]

39. Chapelon-Abric C, de Zuttere D, Duhaut P, Veyssier P, Wechsler B, Huong DLT, et al. Cardiac sarcoidosis: a retrospective study of 41 cases. Medicine (Balt). 2004; 83:315-34.

40. Ridolfi RL, Bulkley BH, Hutchins GM. The conduction system in cardiac amyloidosis: clinical and pathologic features of 23 patients. Am J Med. 1977; 62:677-86. [PubMed: 871125]

41. Reisinger J, Dubrey SW, Lavalley M, Skinner M, Falk RH. Electrophysiologic abnormalities in AL (primary) amyloidosis with cardiac involvement. J Am Coll Cardiol. 1997; 30:1046-51. [PubMed: 9316537]

42. Hancock EW. Cardiomyopathy: Differential diagnosis of restrictive cardiomyopathy and constrictive pericarditis. Heart. 2001; 86:343-9. [PubMed: 11514495]

43. Bongioanni S, Bianchi F, Migliardi A, Gnavi R, Pron PG, Casetta M, et al. Relation of QRS duration to mortality in a community-based cohort with hypertrophic cardiomyopathy. Am J Cardiol. 2007; 100:503-6. [PubMed: 17659936]

44. Persson H, Lonn E, Edner M, Baruch L, Lang CC, Morton JJ, et al. Diastolic dysfunction in heart failure with preserved systolic function: need for objective evidence: results from the CHARM echocardiographic substudy-CHARMES. J Am Coll Cardiol. 2007; 49:687-94. [PubMed: 17291934] 

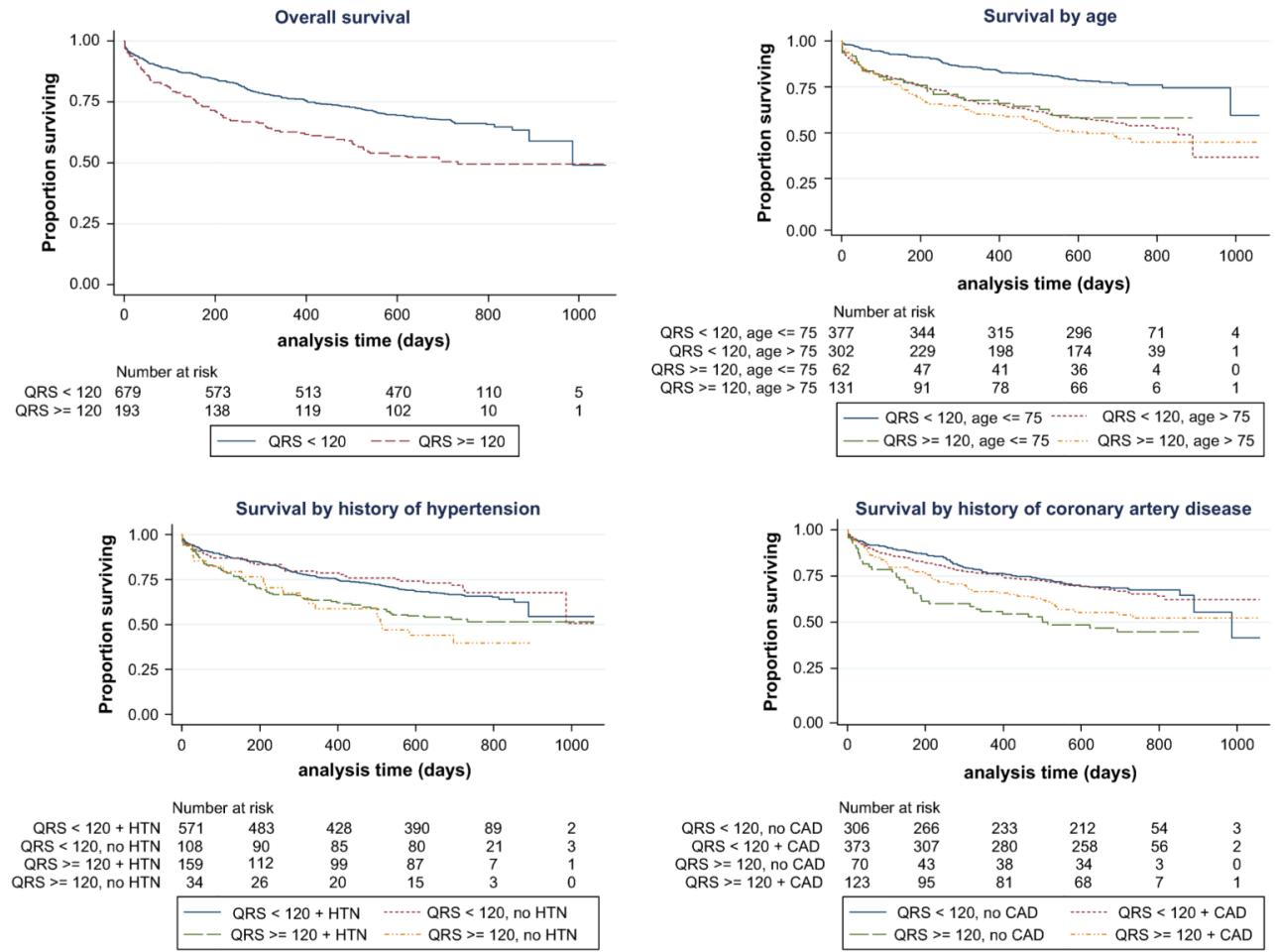

Abbreviations used: QRS, admission EKG QRS duration (in ms); CAD, coronary artery disease; HTN, hypertension

Fig. 1.

Kaplan-Meier survival curves by QRS duration, overall and subgroups. QRS, admission electrocardiogram QRS duration (in ms); CAD, coronary artery disease; HTN, hypertension. 


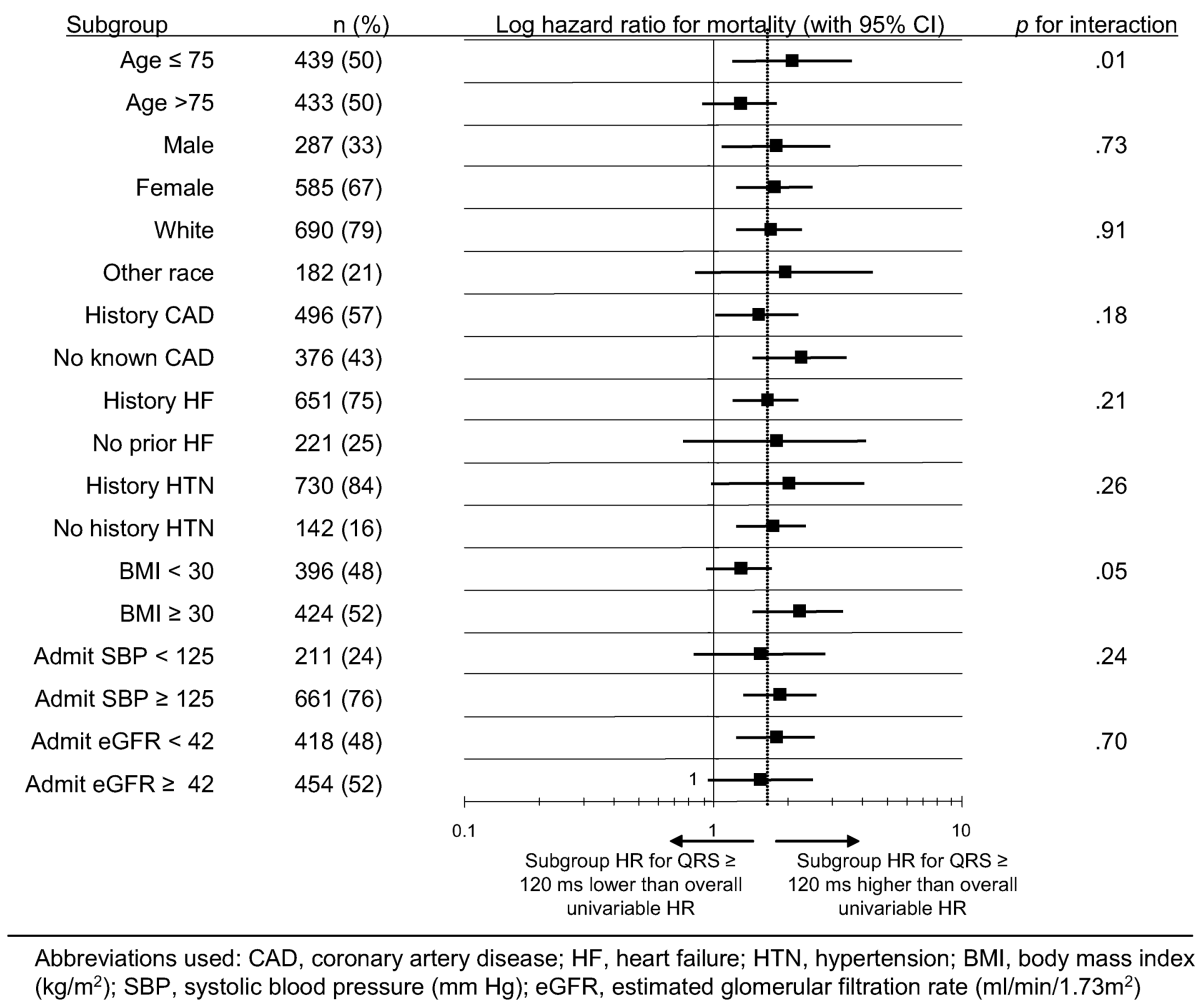

Fig. 2.

Bivariate subgroup analysis for mortality hazard of prolonged ( $>120 \mathrm{~ms}$ ) QRS duration in heart failure with preserved systolic function. CAD, coronary artery disease; HF, heart failure; HTN, hypertension; BMI, body mass index $\left(\mathrm{kg} / \mathrm{m}^{2}\right)$; SBP, systolic blood pressure $(\mathrm{mm} \mathrm{Hg})$; eGFR, estimated glomerular filtration rate $\left(\mathrm{mL} \cdot \mathrm{min} \cdot 1.73 \mathrm{~m}^{2}\right)$. 


\section{Table 1}

Demographic and Clinical Characteristics of Subjects

\begin{tabular}{|c|c|c|c|c|}
\hline Variable & $\begin{array}{c}\text { SHF (overall) } \\
1012)\end{array}$ & $\begin{array}{c}\text { HFPSF (overall) }(\mathbf{n}= \\
\text { 872) }\end{array}$ & $\begin{array}{c}\text { HFPSF }(\text { QRS }<120 \\
\text { ms) }(n=679)\end{array}$ & $\begin{array}{c}\text { HFPSF (QRS } \geq 120 \mathrm{~ms}) \\
(\mathrm{n}=193)\end{array}$ \\
\hline Age (y) & $71 \pm 14$ & $74 \pm 13^{*}$ & $72 \pm 13$ & $78 \pm 11^{\dagger}$ \\
\hline Gender ( $\%$ female) & 41 & $67^{*}$ & 69 & $60^{\dagger}$ \\
\hline Race (\% Caucasian) & 73 & $79^{*}$ & 78 & 82 \\
\hline BMI $\left(\mathrm{kg} / \mathrm{m}^{2}\right)$ & $29.0 \pm 7.6$ & $32.0 \pm 9.4^{*}$ & $32.8 \pm 9.6$ & $29.8 \pm 8.1^{\dagger}$ \\
\hline $\operatorname{LVEF}(\%)$ & $25 \pm 8$ & $60 \pm 8^{*}$ & $60 \pm 8$ & $59 \pm 7$ \\
\hline EFFECT model score (points) & & & $96 \pm 26$ & $105 \pm 26^{\dagger}$ \\
\hline ECG QRS duration (ms) & & & $89 \pm 12$ & $148 \pm 22^{\dagger}$ \\
\hline History of: Heart failure (\%) & 84 & $75^{*}$ & 72 & $83^{\dagger}$ \\
\hline $\mathrm{CAD}(\%)$ & 72 & $57^{*}$ & 54 & $64^{\dagger}$ \\
\hline Atrial fibrillation (\%) & 25 & 27 & 33 & $47^{\dagger}$ \\
\hline Hypertension (\%) & 76 & $84^{*}$ & 84 & 82 \\
\hline Admit systolic BP (mm Hg) & $142 \pm 33$ & $154 \pm 33^{*}$ & $154 \pm 32$ & $157 \pm 33$ \\
\hline Admit diastolic BP (mm Hg) & $79 \pm 21$ & $77 \pm 20^{*}$ & $77 \pm 20$ & $77 \pm 20$ \\
\hline Admit heart rate (beats/min) & $90 \pm 22$ & $85 \pm 22 *$ & $86 \pm 22$ & $84 \pm 21$ \\
\hline Peripheral edema on exam (\%) & 73 & $81^{*}$ & 81 & 79 \\
\hline Radiographic pulmonary edema (\%) & 67 & 70 & 69 & 74 \\
\hline \multicolumn{5}{|l|}{ Laboratory studies at admission: } \\
\hline Serum creatinine & $1.7 \pm 1.4$ & $1.8 \pm 1.7$ & $1.7 \pm 1.6$ & $1.8 \pm 1.9$ \\
\hline Serum sodium & $137 \pm 5$ & $138 \pm 4$ & $138 \pm 4$ & $137 \pm 4^{\dagger}$ \\
\hline Serum hemoglobin & $12.4 \pm 2.0$ & $11.8 \pm 2.0^{*}$ & $11.9 \pm 2.0$ & $11.6 \pm 2.0$ \\
\hline
\end{tabular}

BMI, body mass index; $\mathrm{BP}$, blood pressure; $\mathrm{CAD}$, coronary artery disease; $\mathrm{COPD}$, chronic obstructive pulmonary disease; ECG,

electrocardiogram; HFPSF, heart failure with preserved systolic function; LVEF, left ventricular ejection fraction; SHF, systolic heart failure; EFFECT, Enhanced Feedback for Effective Cardiac Treatment Score.

Continuous variables are presented as means $\pm \mathrm{SD}$.

* Denotes significant $(P<.05)$ difference between SHF and HFPSF in overall population.

${ }^{\dagger}$ Denotes significant $(P<.05)$ difference between HFPSF subjects with QRS <120 ms and QRS $\geq 120 \mathrm{~ms}$. 
Table 2

Univariable and Multivariable Cox Regression for Mortality in HFPSF Patients

\begin{tabular}{lcccc}
\hline Variable & Univariable HR (95\% CI) & $\boldsymbol{P}$ & Adjusted HR (95\% CI) & $\boldsymbol{P}$ \\
\hline ECG QRS duration $\geq 120 \mathrm{~ms}$ & $1.71(1.33-2.19)$ & $<.001$ & $1.31(1.01-1.71)$ & .04 \\
Gender (male vs. female) & $.91(.71-1.16)$ & .44 & $.95(.74-1.23)$ & .72 \\
Race (non-Caucasian vs. Caucasian) & $1.67(1.22-2.28)$ & .001 & $1.21(.87-1.70)$ & .25 \\
EFFECT score (per 10 points) & $1.26(1.21-1.33)$ & $<.001$ & $1.23(1.17-1.29)$ & $<.001$ \\
Heart rate (per 10 beats/minute) & $1.04(.99-1.10)$ & .10 & $1.04(.98-1.09)$ & .18 \\
Prior history of heart failure & $1.97(1.45-2.67)$ & $<.001$ & $1.43(1.02-1.99)$ & .04 \\
Prior history of hypertension & $.98(.72-1.33)$ & .91 & $.87(.63-1.19)$ & .39 \\
Coronary artery disease & $1.02(.81-1.28)$ & .87 & $.79(.62-1.02)$ & .07 \\
Atrial fibrillation & $1.72(1.38-2.16)$ & $<.001$ & $1.33(1.04-1.70)$ & .02 \\
Body mass index $\left(\mathrm{kg} / \mathrm{m}^{2}\right)$ & & & & \\
$\quad<25$ (reference) & 1.0 & & .00 & .04 \\
25 to $<30$ & $.67(.50-.89)$ & .006 & $.72(.53-.98)$ & .04 \\
$\quad 30$ & $.40(.30-.52)$ & $<.001$ & $.53(.40-.70)$ & $<.001$ \\
\hline
\end{tabular}

HR, heart rate; ECG QRS, electrocardiogram QRS interval; the EFFECT score includes the following variables: age, respiratory rate, admission systolic blood pressure, blood urea nitrogen, serum sodium, and admission history of stroke, dementia, chronic obstructive pulmonary disease, hepatic cirrhosis, leukemia, lymphoma, metastatic cancer. 